\title{
THE ESTIMATION OF LINKAGE WITH DIFFERENTIAL VIABILITY
}

\author{
R. A. FISHER and NORMAN T. J. BAILEY \\ Department of Genetics, Cambridge
}

Received 20.viii.48

\section{NOTE ON THE TEST OF SIGNIFICANCE FOR DIFFERENTIAL VIABILITY IN FREQUENCY DATA FROM A COMPLETE THREE-POINT TEST}

\author{
R. A. FISHER
}

IF, in a three-point linkage test, all four triply heterozygous genotypes are used in parallel, the data, after summing complementary genotypes from each cross, take the form of a Latin Square,

TABLE I

Latin square for three-point backcrosses

$\begin{array}{llll}\text { A } & \text { B } & \text { C } & \text { D } \\ \text { B } & \text { A } & \text { D } & \text { C } \\ \text { C } & \text { D } & \text { A } & \text { B } \\ \text { D } & \text { C } & \text { B } & \text { A, }\end{array}$

in which the columns may represent the modes of gamete formation (cross-over classes), the relative frequencies of which it is desired to estimate, the rows may be the four parallel tests, while the letters stand for the four pairs of complementary genotypes obtained in each test.

An overall $\chi^{2}$ test with nine degrees of freedom is, of course, available to detect non-proportionality of the entries in the rows and columns, such as unequal viability of the pairs of genotypes might be expected to produce. We may, however, aim at a more specific test of the three degrees of freedom represented by possibly different viabilities of the four classes of offspring. A simple, though crude, test of this kind was suggested by the author to M. E. Wright, for the test involving sex, wavy and shaker in the sex chromosome of the house mouse. It is the object of the present note to set out an accurate $\chi^{2}$ test for such a case.

Wright's data, including 453 mice bred, are shown in this form below :-

TABLE 2

Data for wavy, shaker and sex

\begin{tabular}{|c|c|c|c|c|c|}
\hline & & mbin & nvolv & & \\
\hline & None & Sex & $w v_{2}$ & $s h_{2}$ & Total \\
\hline $\begin{array}{l}\text { All in coupling } \\
w v_{2}-s h_{2} \text { in coupling } \\
s h_{2} \text {-sex in coupling } \\
w v_{2} \text {-sex in coupling }\end{array}$ & $\begin{array}{r}23 \\
36 \\
39 \\
29\end{array}$ & $\begin{array}{l}44 \\
55 \\
46 \\
40\end{array}$ & $\begin{array}{l}18 \\
17 \\
19 \\
15\end{array}$ & $\begin{array}{l}21 \\
12 \\
23 \\
16\end{array}$ & $\begin{array}{l}106 \\
120 \\
127 \\
100\end{array}$ \\
\hline$\underset{215}{\text { Total }}$ & . 127 & 185 & 69 & 72 & $2^{453}$ \\
\hline
\end{tabular}


From the marginal totals proportionate expectations are calculated for each cell, as in the table following :-

TABLE 3

Expectations for equal viability

\begin{tabular}{cccc|c}
$29 \cdot 7174$ & $43 \cdot 2892$ & $16 \cdot 1457$ & $16 \cdot 8477$ & $106 \cdot 0000$ \\
$33 \cdot 6424$ & $49 \cdot 0066$ & $18 \cdot 271$ & $19 \cdot 0729$ & $120 \cdot 0000$ \\
35.6049 & $51 \cdot 8653$ & $19 \cdot 3444$ & $20 \cdot 1854$ & $127 \cdot 0000$ \\
$28 \cdot 0353$ & $40 \cdot 8389$ & $15 \cdot 2318$ & $15 \cdot 8940$ & $100 \cdot 0000$ \\
\hline $127 \cdot 0000$ & $185 \cdot 0000$ & $69 \cdot 0000$ & $72 \cdot 0000$ & $453 \cdot 0000$
\end{tabular}

From these, comparing each frequency separately with its expectation we find

$$
x^{2}=7 \cdot 8239
$$

for nine degrees of freedom, giving no indication of disturbances due to unequal viability.

The simple test referred to above consists in comparing the total expectations for each pair of genotypes derived from this table, with the totals observed, thus :-

TABLE 4

Crude test for viability

\begin{tabular}{cccc} 
Pair of genotypes & Expected & Observed & Deviation \\
A & 113.9624 & 113 & -0.9624 \\
B & 112.3488 & 118 & +5.6512 \\
C & 111.6624 & 109 & -2.6624 \\
D & 115.0264 & 113 & -2.0264 \\
\hline Total & 453.0000 & 453 & 0.0000
\end{tabular}

giving a value

$$
\chi^{2}=0.39157
$$

for three degrees of freedom. This is exceptionally small, and leaves no ground for suspecting any viability disturbance. Its very smallness, however, arouses some suspicion that it may be theoretically invalid, and indeed on reflection it appears likely that some part of the chance variations in numbers of the different pairs of genotypes may have been compensated in adjusting the expectations from the totals of the rows and columns.

The exact treatment of the problem in which the frequencies are affected by arbitrary viability differences, and in which the expectations are therefore the products of three unknown factors, offers exceptional algebraic difficulty. An exact test of significance for disturbance of viability, which a priori is always to be suspected in such data, is, however, a real need, so that without elaborate algebraic discussion it may be useful to set out such a test, and to illustrate it on these data. 
Representing the column totals by $p, q, r, s$ and the row totals by $a, b, c, d$ out of a grand total of $\mathcal{N}$ observations we may calculate the matrix

$\frac{16}{\mathcal{N}^{3}}\left\{\begin{array}{ccc}(a+b)(c+d)(p+q)(r+s) & (a d-b c)(p s-q r) & (a c-b d)(p r-q s) \\ (a d-b c)(p s-q r) & (a+c)(b+d)(p+r)(q+s) & (a b-c d)(p q-r) \\ (a c-b d)(p r-q s) & (a b-c d)(p q-r s) & (a+d)(b+c)(p+s)(q+r)\end{array}\right\}$

having the numerical values :-

TABLE 5

Covariance matrix of viability scores

$\begin{array}{rrr}388 \cdot 44844 & 2 \cdot 89182 & -I \cdot 14671 \\ 2 \cdot 89182 & 444 \cdot 41957 & \cdot 06378 \\ -1 \cdot 14671 & \cdot 06378 & 44^{2} \cdot 66618\end{array}$

This is the covariance matrix, subject to fixed marginal totals, of scores for parameters of differential viability derivable from the deviations in table 4 , as follows :-

$$
\begin{array}{lr}
A+B-C-D & 9 \cdot 3776 \\
A-B+C-D & -7 \cdot 2496 \\
A-B-C+D & -5.9776
\end{array}
$$

Inverting the matrix to supply multipliers, and for convenience multiplying by 100o, we have :-

TABLE 6

Covariance matrix for estimating

$\begin{array}{rrr}2 \cdot 5744887 & -\cdot 0167530 & \cdot 0066715 \\ -.0167530 & 2 \cdot 2502350 & -0003676 \\ .0066715 & -.0003676 & 2 \cdot 2590560\end{array}$

The sums of the products of the scores with the corresponding rows of any column now give estimates of viability differences corresponding with the scores, and the sum of the products of scores and estimates supplies the $\chi^{2}$ needed for testing significance

with

$\begin{array}{crr}\text { Estimate of viability deviation } & \text { Score } \\ \lambda & \cdot 024224 & 9.3776 \\ \mu & -\cdot 016468 & -7.2496 \\ \nu & -.013439 & -5.9776\end{array}$

$$
\chi^{2}=0.42688
$$

for three degrees of freedom.

Wright's conclusion that the viability disturbances are negligible is thus confirmed by a more exact analysis. The value of $\chi^{2}$ is only a trifle greater than before.

The estimates obtainable by this method evidently supply material for a first correction of the column factors appropriate to small but significant differences in viability. I am not satisfied how this can best be done, and the present example, with no sign of genuine viability disturbance, is not suitable for exhibiting the methods that might be used. 
As a first step, however, we may use as natural logarithms of the estimated viability factors, the expressions
A
$\lambda+\mu+\nu$
$-\cdot 005683$
B
$\lambda-\mu-v$
$+\cdot 054131$
C
$-\lambda+\mu-\nu$
$-\lambda-\mu+\nu$
$-.027253$
D$$
-.021195
$$

with corresponding increments of the natural logarithms of the column factors

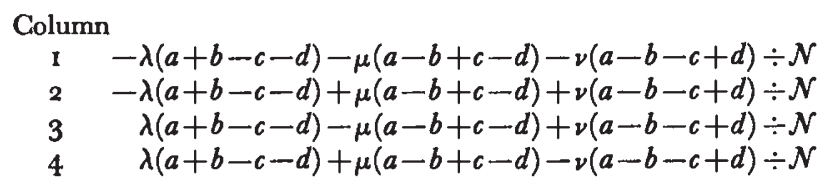

and for the row factors

$$
\begin{array}{cl}
\text { Row } & \\
1 & -\lambda(p+q-r-s)-\mu(p-q+r-s)-\nu(p-q-r+s) \div \mathcal{N} \\
2 & -\lambda(p+q-r-s)+\mu(p-q+r-s)+\nu(p-q-r+s) \div \mathcal{N} \\
3 & -\lambda(p+q-r-s)-\mu(p-q+r-s)+\nu(p-q-r+s) \div \mathcal{N} \\
4 & -\lambda(p+q-r-s)+\mu(p-q+r-s)-\nu(p-q-r+s) \div \mathcal{N}
\end{array}
$$

The adjustments in the column factors vanish if the row totals $a, b, c$ and $d$ are all equal, the ideal condition in the absence of differential viability, and are small if they are nearly equal, as in the example. Numerically, for the four columns they are :-

TABLE 7

Logarithmic adjustments of column factors

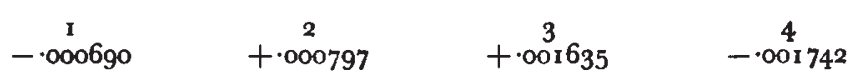

Increasing the natural logarithms of the observed totals by these amounts, adding and dividing by the total, we have percentage estimates of the frequencies of the four modes of gamete formation, agreeing closely with those obtained without adjustment, i.e. :-

TABLE 8

Frequencies of modes of gamete formation

Recombination
involving
None
Sex
$w v_{2}$
$s h_{2}$

\begin{tabular}{cc}
$\begin{array}{c}\text { Without } \\
\text { adjustment }\end{array}$ & $\begin{array}{c}\text { Adjusted for } \\
\text { viability }\end{array}$ \\
$28 \cdot 0353$ & $28 \cdot 0130$ \\
$40 \cdot 8389$ & $40 \cdot 8671$ \\
$15 \cdot 2318$ & $15 \cdot 2551$ \\
$15 \cdot 8940$ & $15 \cdot 8647$ \\
\hline $100 \cdot 0000$ & $99 \cdot 9999$
\end{tabular}

as must equally be the case with the recombination estimates :-

TABLE 9

Recombination fractions

Without adjustment

percentage

$w 0_{2}-s h_{2}$
$w v_{2}-\operatorname{sex}$
$s h_{2}-\operatorname{sex}$
$31 \cdot 1258$

$56 \cdot 0707$

$56 \cdot 7329$
Adjusted for viability

percentage

$31 \cdot 1198$

$5^{6} \cdot 1222$

$56 \cdot 7318$ 
The only perceptible change is a slight rise of about one-twentieth of I per cent. in the estimated recombination fraction of wavy with sex. It is evident that the excess of this value above $5^{\circ}$ per cent. is not to be explained by anything in the nature of viability differences among the genotypes.

Finally, that this method, while not providing a perfect fit, goes some way towards doing so may be seen by reconstructing the whole table of expectations, using the logarithmic adjustments explained above. We find then :-

TABLE IO

Adjusted expectations

\begin{tabular}{cccc|r}
$29 \cdot 1310$ & $45 \cdot 1176$ & 15.5254 & $16 \cdot 2439$ & $106 \cdot 0179$ \\
35.2822 & $48 \cdot 4833$ & 17.8195 & $18 \cdot 4197$ & $120 \cdot 0047$ \\
34.9003 & $51 \cdot 2239$ & $19 \cdot 4201$ & $21 \cdot 4410$ & 126.9853 \\
$27 \cdot 6801$ & $40 \cdot 1376$ & $16 \cdot 2522$ & $15 \cdot 9212$ & $99 \cdot 9921$ \\
\hline 126.9936 & 184.9624 & $69 \cdot 0182$ & $72 \cdot 0258$ & $453 \cdot 0000$
\end{tabular}

The aim of the viability adjustments has been to bring the expected totals for genotypes into agreement with the numbers observed. The extent to which this has been accomplished is shown below :-

TABLE II

Frequencies of pairs of complementary genotypes

$\begin{array}{cccc}\text { Unadjusted } & \begin{array}{c}\text { Observed } \\ \text { frequency }\end{array} & \begin{array}{c}\text { Adjusted } \\ \text { expectation }\end{array} \\ \text { A } & 113.9624 & 113 & 112.9556 \\ \text { B } & 112.3489 & 118 & 118.0940 \\ \text { C } & 111.6624 & 189 & 108.9830 \\ \text { D } & 115.0264 & 113 & 112.9674\end{array}$

The largest discrepancy has been reduced about sixty-fold, and this without introducing any crying discrepancy in the totals for rows and columns. Generally speaking the viability corrections appear to have been slightly in excess of what was needed.

Obviously, in this example, the correction of viability disturbances, which are far from significant, is of no practical value. The method, however, seems to be serviceable, in the absence of a more exact approach, in cases in which small though real viability differences are suspected. 